\title{
Forecasting Cyclical and Non-cyclical Stock Prices on the Stock Exchange of Thailand
}

\author{
Rapin Sunthornwat ${ }^{a}$, Yupaporn Areepong ${ }^{\mathrm{b}, *}$ \\ a Industrial Technology Program, Faculty of Science and Technology, Pathumwan \\ Institute of Technology, Bangkok, 10330, Thailand; b Department of Applied Statistics, \\ Faculty of Applied Science, King Mongkut's University of Technology North Bangkok, \\ Bangkok, 10800, Thailand.
}

*For correspondence: yupaporn.a@sci.kmutnb.ac. th

Received: 1 March 2021

Accepted: 15 October 2021

(C) Copyright Sunthornwat.

This article is distributed

under the terms of the

Creative Commons

Attribution License, which

permits unrestricted use

and redistribution provided

that the original author and

source are credited.
Abstract Forecasting is an important role in organizations for decision making and planning. This research is to forecast the cyclical and non-cyclical weekly stock prices on the Stock Exchange of Thailand by using the models of Geometric Brownian motion, Fourier's series, and Cauchy initial value problem. The accuracy and performance of the models are based on the minimum root mean squared percentage error which is the error between actual and forecasted stock prices. The results showed that Geometric Brownian motion is suitable for forecasting both cyclical and non-cyclical stock prices because of minimum error. Moreover, the confidence intervals of forecasted stock prices are demonstrated. Therefore, Geometric Brownian motion should be selected to describe the movement of stock prices in Thailand.

Keywords: Forecasting, Geometric Brownian motion, Fourier's series, Cauchy initial value problem.

\section{Introduction}

One of the many problems which involves making a decision is forecasting. The forecasting was employed in what the outcome is in the future. The good performance of forecasting leads to the good performance of profitability of the organization. The organization which can forecast future events will be able to manage uncertain future events better than the organization with unknown information in the future. Several processes in an organization even individually still require forecasting in that it is the most accurate in order to improve and analyze the processes. Namely, forecasting has widely been used in such processes as the production process, inventory process, marketing process, personal financial process, etc. Thus, forecasting is an important key of a successful organization and person. In particular, forecasting the price of risky assets which is an uncertainty of price movement. For example, the organization needs the most forecasting techniques for forecasting the food of animal in the future on future contracts and options so as to plan the animal food to be enough in case of lacking production corp. Investors try to simulate and forecast the movement of risky assets for capital gain. They need accurate forecasting techniques for simulating the movement of the risky asset.

A lot of research has been studied about forecasting techniques. One of the best methods for forecasting is Box-Jenkins technique (see Box and Jenkins [1]) based on the linear relation among random variables with lagging time. Auto-Regressive Integrated Moving Average (ARIMA) models of the Box-Jenkins technique is a mathematical model for representing the forecasting on time series. Later, the extension of the ARIMA model has been used to apply in many fields. The next-day electricity prices using ARIMA models on the spot market of mainland Spain and Californian were forecasted [2]. The ARIMA model to 
forecast commodity prices was employed [3]. The emission of Carbon Dioxide in Bangladesh using ARIMA $(0,2,1)$ model which is the best fitted model was modeled and forecasted [4]. ARIMA $(1,2,1)$ for forecast the annual total Carbon Dioxide in China. The trend of Carbon Dioxide in China is likely to increase was constructed [5]. The forecasting model for curly red chili price at retail level using ARIMA $(1,1,9)$ model was constructed [6]. ARIMA $(1,1,2)$ model to forecast monthly malaria incidence between 2018 and 2019 in Kumasi Metropolis was used [7]. The monthly price in selected markets from April 2000 to December 2014 of Bengalgram using ARIMA $(1,1,1)$ model was forecasted [8]. Meanwhile, the data with high volatility have been often seen in real applications. Several researchers have been studied in combination between ARIMA model and Generalised AutoRegressive Conditional Heteroskedasticity (GARCH) model on high volatility real data especially financial data. The volatility of gold future contract (GF10J16) using ARIMAGARCH model based on the gold future price movement followed normal, $t$, and generalized error distributions and compare cumulative of return of ARIMAGARCH model was forecasted [9]. The machine health condition generated from vibrated signal to explain the condition of the wear and fault on the machine using ARMAGARCH was estimated and forecasted [10]. The ARMAGARCH model to forecast mean and volatility in short-term (hourly, weekly, monthly) of electricity prices was applied [11]. The network traffic based on the ARIMAGARCH model for capturing the traffic characteristics and traffic network controlling was predicted [12]. The proposed model has better accuracy of forecasting than fuzzy autoregressive integrated moving average model. Oil prices and volatility of the returns of oil prices using Hybrid ARIMAGARCH model were forecasted. Hybrid ARIMA $(33,0,14)$ GARCH $(1,2)$ model based on dynamic and static procedure is suitable for the returns of oil prices. The static procedure was better performance than dynamic procedure [13]. In addition, combined models between ARIMA models and artificial intelligence models such as Neural Networks model, Support vector machines, etc. have been widely used for forecasting. ARIMA model and Support vector machines which is a new artificial neural network technique to model the stock price were combined [14]. A hybrid model between ARIMA and neural networks to forecast the real data was used. The combined model between ARIMA and artificial neural network technique performed better than the single model, ARIMA or artificial neural network model [15]. ARIMA and a fuzzy regression model for forecasting the exchange rate of NT dollars to US dollars were combined [16]. The commodity of the future trend of Iran's crude steel consumption using fuzzy ARIMA model which provided significantly improved accuracy was forecasted [17]. Fuzzy seasonal ARIMA forecasting short - term model which is a combined model between fuzzy and seasonal ARIMA model for forecasting time series data of Taiwan machinery industry and the soft drink was presented [18]. As mentioned in the above research works, it found that the general time series data is the most selected to be the samples of the research works. The most forecasting models are based on ARIMA or combination between ARIMA model and artificial intelligent model which are suitable for linear pattern of time series data.

This research is focused on the random and periodic models -- Geometric Brownian motion, Fourier's series, and Cauchy initial value problem-- for forecasting cyclical time series data on the Stock Exchange of Thailand. However, time series data with cyclical patterns specifically stock price movement on the Stock Exchange of Thailand is sampled as the sample of this research. It is important for investors to find a suitable chance in taking profit and capital gain on the investment if there is the best model for describing and forecasting the time series data movement or stock price movement. Therefore, cyclical stock price as cyclical time series data is an interesting issue for studying its price movement and forecasting of cyclical stock price. The stock price samples of both non-cyclical and cyclical stocks of this study are selected by the Stock Exchange of Thailand. Also, the non-cyclical stock price is brought to compare the methods for this research with the cyclical stock price.

\section{Materials and methods}

In this section, some fundamental knowledge and background about the characteristics of cyclical stocks and non-cyclical stocks are described as the materials for this research (see [19]). In addition, the methods for forecasting the stock prices: geometric Brownian motion (see [20]), Fourier's series (see [21]), and Cauchy initial value problem (see [22]) are shown as follows: 


\section{Materials}

\section{Cyclical and Non - cyclical Stock Price}

Business cycle is a change of a business by the changing of economic factors which affect the stock prices. The business cycle is influenced by the external and internal economic factors of the economic system. The range of time in the business cycle depends on the strength of the global economy. In addition, the business cycle consists of 4 stages as follows:

1. Expansion is an increase in the range of economic factors such as production, employment, revenue. The direction of the investment is in an increasing trend.

2. Boom is the range which is increased by the strategic planning. It is a maximum point of the business cycle. The economic factors in particular the production cost and the price of finished goods are also the highest.

3. Recession is the range in which the investment direction decreases. The demand for finished goods and employment are also in the decrease.

4. Depression is the range which is in the minimum point of the business cycle. The economic system is a contraction such as low production and high unemployment.

5.

Thus, the stock prices which depend on the business cycle are called cyclical stock (sometimes called offensive stock or aggressive stock) price; otherwise non-cyclical stock (sometimes called defensive stock) prices. Namely, the cyclical stock is the stock which is influenced by the economic cycle. The price movement of the cyclical stock depends on the movement of economic expansion and economic depression. This phenomenon makes a cycle on the stock price. In general, the cyclical stock has the behavior of its stock price movement in up and down followed by the business cycle such as automobile stock price which is a non-essential goods for consumers. On the other hand, the non-cyclical stock has the behavior of its stock price independent movement because its business company produces the essential goods for consumers such as food stock price which is a necessary commodity for consumers even during the depression economic period. Thus cyclical stock is opposite of non-cyclical stock. For some examples on the Stock Exchange of Thailand, some cyclical stocks consist of: construction materials company, paper and printing materials company, industrial tool and machinery company, vehicle and equipment company, automobile company, airline company, jewelry company, etc., but some non-cyclical stocks consist of: public utility company, infrastructure company, beverage company, food company, medical company, etc.

Thus, the cyclical stock is a risky stock in the point of view of many investors because the Beta $(\beta)$ value is higher than non-cyclical stock. The Beta value is a statistic on the Capital Asset Pricing Model (CAPM) for measuring the risk of stock with respect to the total market value with each other's linear relationship. For this research, the CAPM is an important model for studying the linear relationship between the return on individual stock and the return on the Stock Exchange of Thailand index. The Beta value of CAPM model can be used for evaluation of the risk and the return of individual stock with respect to the Stock Exchange of Thailand. If the Beta value of individual stock is high, then the individual stock will be high risk and high return. It can be defined as

$$
\mathbf{E}\left(r_{s}\right)=r_{f}+\left[\mathbf{E}\left(r_{M}\right)-r_{f}\right] \beta
$$

where $\mathbf{E}\left(r_{s}\right)$ is an expectation return on risky stock price,

$\mathbf{E}\left(r_{M}\right)$ is an expectation return on market value,

$r_{f}$ is a return on risk free assets.

If the Beta value equals 1 then the return on market value increases (decreases) $1 \%$ and the return of risky stock price will also increase (decrease) $1 \%$. That is, the risky stock equally risks the market. If the Beta value is greater than 1 then the return on market value increases (decreases) $1 \%$ and the return of risky stock price will also increase (decrease) more than $1 \%$. If the Beta value is less than 1 then the return on market value increases (decreases) $1 \%$ and the return of risky stock price will also increase (decrease) less than $1 \%$.

\section{Drift and Volatility of Stock Price}

Let $S_{t}$ be a stock prices at time $t$. The expected value and variance of stock prices are calculated on the return which $r_{i}$ is defined as 
$r_{t}=\frac{S_{t+1}-S_{t}}{S_{t}} ; t=1,2,3, \ldots, n$.

Drift of stock prices is defined as

$\mu=\frac{1}{n \Delta t} \sum_{t=1}^{n} r_{t}$

and volatility of stock prices is defined as

$\sigma=\sqrt{\frac{1}{(n-1) \Delta t} \sum_{t=1}^{n}\left(r_{t}-\bar{r}\right)^{2}}$

\section{Geometric Brownian Motion}

Let $S_{0}$ be an initial state of the stock price at time $t=0$. The stock price $S_{t}$ is a random variable at time $t=1,2,3, \ldots, n$. Given $t_{i}=i \Delta t$ and $\Delta t \rightarrow 0$, the model for stock price is moved by the discrete model as

$$
S\left(t_{i+1}\right)=S\left(t_{i}\right)+\mu \Delta t S\left(t_{i}\right)+\sigma \sqrt{\Delta t} Y_{i} S\left(t_{i}\right)
$$

where $\mu$ is a drift of stock price,

$\sigma$ is a volatility of stock price,

$Y_{0}, Y_{1}, Y_{2}, \ldots$ is an i.i.d. $N(0,1)$.

For continuous model, the time interval $[0, t]$ and $t=k \Delta t$ for $k=1,2,3, \ldots, L$ are considered. From discrete model on Equation (2), $S(\Delta t), S(2 \Delta t), \ldots, S(L \Delta t=t)$ are evaluated. As $\Delta t \rightarrow 0$ and $L \rightarrow \infty$, discrete model transformed into continuous model as

$S(t)=S_{0} \prod_{i=0}^{L-1}\left(1+\mu \Delta t+\sigma \sqrt{\Delta t} Y_{i}\right)$

Taking logarithm on both sides,

$$
\log \left(\frac{S(t)}{S_{0}}\right)=\sum_{i=0}^{L-1} \log \left(1+\mu \Delta t+\sigma \sqrt{\Delta t} Y_{i}\right)
$$

From Taylor's series of $\log (1+x) \approx x-\frac{x^{2}}{2}+\ldots$ with small $x$, Equation (3) becomes

$$
\log \left(\frac{S(t)}{S_{0}}\right) \approx \sum_{i=0}^{L-1}\left(\mu \Delta t+\sigma \sqrt{\Delta t} Y_{i}-\frac{1}{2} \sigma^{2} \Delta t Y_{i}^{2}\right)
$$

where $\mathbf{E}\left(\mu \Delta t+\sigma \sqrt{\Delta t} Y_{i}-\frac{1}{2} \sigma^{2} \Delta t Y_{i}^{2}\right)=\mu \Delta t-\frac{1}{2} \sigma^{2} \Delta t$,

$$
\operatorname{var}\left(\mu \Delta t+\sigma \sqrt{\Delta t} Y_{i}-\frac{1}{2} \sigma^{2} \Delta t Y_{i}^{2}\right)=\sigma^{2} \Delta t+\text { higher term of } \Delta t
$$

From the Central Limit Theorem, term $\log \left(\frac{S(t)}{S_{0}}\right)$ in Equation (4) follows the normal random variable with mean $L\left(\mu \Delta t-\frac{1}{2} \sigma^{2} \Delta t\right)=\left(\mu-\frac{1}{2} \sigma^{2}\right) t$ and variance $L \sigma^{2} \Delta t=\sigma^{2} t$. Namely,

$$
\log \left(\frac{S(t)}{S_{0}}\right) \sim N\left(\left(\mu-\frac{1}{2} \sigma^{2}\right) t, \sigma^{2} t\right)
$$

Thus, the stock prices at time $t$ become

$$
S(t)=S_{0} e^{\left(\mu-\frac{1}{2} \sigma^{2}\right) t+\sigma \sqrt{t} Z} \quad \text { where } Z \sim N(0,1),
$$

and the stock price from time $t=t_{1}$ to $t=t_{2}$ where $t_{2}>t_{1}$ as 
$\log \left(\frac{S\left(t_{2}\right)}{S\left(t_{1}\right)}\right) \sim N\left(\left(\mu-\frac{1}{2} \sigma^{2}\right)\left(t_{2}-t_{1}\right), \sigma^{2}\left(t_{2}-t_{1}\right)\right)$

Because $Y_{i}$ in Equation (2) is the i.i.d. random variable,

$$
\log \left(\frac{S\left(t_{3}\right)}{S\left(t_{2}\right)}\right) \sim N\left(\left(\mu-\frac{1}{2} \sigma^{2}\right)\left(t_{3}-t_{2}\right), \sigma^{2}\left(t_{3}-t_{2}\right)\right) \text { for } t_{3}>t_{2}>t_{1}
$$

and is independent with $\log \left(\frac{S\left(t_{2}\right)}{S\left(t_{1}\right)}\right)$.

Therefore, for $0=t_{0}<t_{1}<t_{2}<\ldots<t_{M}$, the stock prices can be described on the model as

$$
S\left(t_{i+1}\right)=S\left(t_{i}\right) e^{\left(\mu-\frac{1}{2} \sigma^{2}\right)\left(t_{i+1}-t_{i}\right)+\sigma \sqrt{t_{i+1}-t_{i}} Z_{i}}
$$

where i.i.d. $Z_{i} \sim N(0,1)$.

\section{Fourier's Series}

The concept of Fourier's series is to estimate given function by using a periodic trigonometric function. Namely, the origin of continuous Fourier's series is generated by the convergent trigonometric series of the form for estimating $f(t)$ :

$$
\begin{aligned}
f(t)= & a_{0}+a_{1} \cos (t)+a_{2} \cos (2 t)+\ldots+a_{N} \cos (N t)+\ldots \\
& +b_{1} \sin (t)+b_{2} \sin (2 t)+\ldots+b_{N} \sin (N t)+\ldots
\end{aligned}
$$

where $a_{0}, a_{1}, a_{2}, \ldots, a_{N}, \ldots$ and $b_{0}, b_{1}, b_{2}, \ldots, b_{N}, \ldots$ are called the coefficients of Fourier's series for some positive integer $N$. Here, these coefficients are real.

The above series can be rewritten on $\left[-\frac{L}{2}, \frac{L}{2}\right]$ as

$$
f(t)=a_{0}+\sum_{n=1}^{\infty}\left[a_{n} \cos \left(\frac{2 n \pi t}{L}\right)+b_{n} \sin \left(\frac{2 n \pi t}{L}\right)\right]
$$

where

$$
\begin{aligned}
& a_{0}=\frac{1}{L} \int_{-\frac{L}{2}}^{\frac{L}{2}} f(t) d t, \\
& a_{n}=\frac{2}{L} \int_{-\frac{L}{2}}^{\frac{L}{2}} f(t) \cos \left(\frac{2 n \pi t}{L}\right) d t, \\
& \text { and } b_{n}=\frac{2}{L} \int_{-\frac{L}{2}}^{\frac{L}{2}} f(t) \sin \left(\frac{2 n \pi t}{L}\right) d t .
\end{aligned}
$$

For discrete Fourier's series, the concept on the substitution of summation to integral is adopted to derive the discrete case. Taking the summation on both side of Equation (7),

$$
\begin{aligned}
& \sum_{t=1}^{k} f(t)=\sum_{t=1}^{k} a_{0}+\sum_{t=1}^{k} \sum_{n=1}^{N}\left[a_{n} \cos \left(\frac{2 n \pi t}{L}\right)+b_{n} \sin \left(\frac{2 n \pi t}{L}\right)\right] \\
& \sum_{t=1}^{k} f(t)=a_{0}(k)+\sum_{n=1}^{N}\left[a_{n} \sum_{t=1}^{k} \cos \left(\frac{2 n \pi t}{L}\right)+b_{n} \sum_{t=1}^{N} \sin \left(\frac{2 n \pi t}{L}\right)\right]
\end{aligned}
$$

Thus,

$$
a_{0}=\frac{1}{k} \sum_{t=1}^{k} f(t)
$$


$\sum_{t=1}^{k} f(t) \cos \left(\frac{2 n \pi t}{L}\right)=\sum_{t=1}^{k} a_{0} \cos \left(\frac{2 n \pi t}{L}\right)+\sum_{t=1}^{k} \sum_{n=1}^{N}\left[a_{n} \cos \left(\frac{2 n \pi t}{L}\right)+b_{n} \sin \left(\frac{2 n \pi t}{L}\right)\right] \cos \left(\frac{2 n \pi t}{L}\right)$

Thus,

$a_{n}=\frac{2}{k} \sum_{t=1}^{k} f(t) \cos \left(\frac{2 n \pi t}{L}\right)$

$\sum_{t=1}^{k} f(t) \sin \left(\frac{2 n \pi t}{L}\right)=\sum_{t=1}^{k} a_{0} \sin \left(\frac{2 n \pi t}{L}\right)+\sum_{t=1}^{k} \sum_{n=1}^{N}\left[a_{n} \cos \left(\frac{2 n \pi t}{L}\right)+b_{n} \sin \left(\frac{2 n \pi t}{L}\right)\right] \sin \left(\frac{2 n \pi t}{L}\right)$

Thus,

$b_{n}=\frac{2}{k} \sum_{t=1}^{k} f(t) \sin \left(\frac{2 n \pi t}{L}\right)$

Ordinary differential equations of Cauchy initial value problems

The Cauchy initial value problem is an ordinary differential equation which is formed as

$$
\frac{d y}{d t}=a y, \quad y\left(t_{0}\right)=y_{0}
$$

The method for solving Equation (8) is based on the methods given by Lascsáková (see [22]). The particular solution solved from Equation (8) is

$$
y=y_{0} e^{a\left(t-t_{0}\right)}
$$

For any point $\left(t_{i}, y_{i}\right)$, Equation (9) can rewrite and a can be evaluated as

$$
y_{i+1}=y_{i} e^{a\left(t_{i+1}-t_{i}\right)}
$$

where $a=\frac{1}{t_{i}-t_{i-1}} \ln \left(\frac{y_{i}}{y_{i-1}}\right)$.

However, the solution as in Equation (10) should specifically be agreement with the real data which be growth as exponential function. Lascsáková (see [22]) extended the numerical method for solving Equation (8) with assumptions in that the data possesses trend and exponential growth as.

$t_{i+1}=t_{i}+h$

$y_{i+1}=y_{i}+b h+Q e^{v t_{i}}\left(e^{v h}-1\right)$, for $i=1,2,3, \ldots$

where $h=t_{i+1}-t_{i}$ is a step size,

$$
\begin{aligned}
& f\left(t_{i}, y_{i}\right)=a y_{i}, \\
& f^{\prime}\left(t_{i}, y_{i}\right)=a y^{\prime}\left(t_{i}\right)=a^{2} y_{i}, \\
& f^{\prime \prime}\left(t_{i}, y_{i}\right)=a^{2} y^{\prime}\left(t_{i}\right)=a^{3} y_{i}, \\
& v=\frac{f^{\prime \prime}\left(t_{i}, y_{i}\right)}{f^{\prime}\left(t_{i}, y_{i}\right)} \\
& Q=\frac{f^{\prime}\left(t_{i}, y_{i}\right)-f^{\prime \prime}\left(t_{i}, y_{i}\right)}{(1-v) v^{2} e^{v t_{i}}}, \\
& b=f\left(t_{i}, y_{i}\right)-\frac{f^{\prime}\left(t_{i}, y_{i}\right)}{v} .
\end{aligned}
$$




\section{Methods}

\section{Data Collection}

Data for this research was gathered from the Stock Exchange of Thailand. The weekly data at close price can be classified into two groups: cyclical stock and non-cyclical stock from 13/12/2004 to $10 / 02 / 2020$. Additionally, in sample starts at $13 / 12 / 2004$ to $02 / 01 / 2017$ and out of sample starts at 09/01/2017 to $10 / 02 / 2020$.

The backgrounds of the sample companies as the cyclical stocks consist of the followings.

1. $\mathrm{CH}$. Karnchang Public Company Limited (stock name: $\mathrm{CK}$ ) is the Construction Services sector in The Stock Exchange of Thailand. The company is a developer of basic infrastructure and operation of construction business.

2. Italian-Thai Development Corporation Limited (stock name: ITD) is the construction services sector in The Stock Exchange of Thailand. The company is also a developer of basic infrastructure which originated by Thai and Italian.

3. Nawarat Patanakarn Public Company Limited (stock name: NWR) is the construction services sector in The Stock Exchange of Thailand. The company operates its business as construction contractor, manufacturer, and joint venture.

4. Thoresen Thai Agencies Public Company Limited (stock name: TTA) is a transportation and logistics company in The Stock Exchange of Thailand. The company operates its business in shipping, offshore service, agrochemical, and investment.

The backgrounds of the sample companies as the non-cyclical stocks consist of the followings

1. Bangkok Hospital (stock name: BDMS) is a health care service in The Stock Exchange of Thailand. The aims of the company to provide medical services with warmth and completion.

2. CP All Public Company Limited (stock name: CPALL) is a commerce sector in The Stock Exchange of Thailand. The company operates its business in convenience stores.

3. Charoen Pokphand Foods Public Company Limited (stock name: CPF) is the food and beverage sector in The Stock Exchange of Thailand. The company is a group of companies which operates its business in an agro-industrial and food industry.

4. Thai Union Group Public Company Limited (stock name: TU) is the food and beverage sector in The Stock Exchange of Thailand. Its business is operated on frozen and canned seafood.

\section{Computation of forecasting the stock prices}

In this section, the procedures for forecasting some stock prices: cyclical and non-cyclical on the Stock Exchange of Thailand are proposed as follows:

Procedure 1: forecasting the stock prices by Geometric Brownian motion Let drift and volatility of stock prices be $\mu$ and $\sigma$ respectively on time interval $[0, T]$.

Step 1: Calculate initial values

$i=0$,

$\Delta t=\frac{T}{n}=t_{i+1}-t_{i}$,

$S\left(t_{0}\right)=\operatorname{Max}\left\{S\left(t_{i}\right): t_{i} \in\left[0, i \Delta t_{i}\right]\right.$,

$\operatorname{Max}\left\{t_{i}: t_{i} \in\left[0, i \Delta t_{i}\right]\right\}=0$

$r\left(t_{i}\right)=\frac{S\left(t_{i+1}\right)-S\left(t_{i}\right)}{S\left(t_{i}\right)}$

$\mu=\frac{1}{n \Delta t} \sum_{t=1}^{n} r\left(t_{i}\right)$

$\sigma=\sqrt{\frac{1}{(n-1) \Delta t} \sum_{t_{i}=1}^{n}\left[r\left(t_{i}\right)-\overline{r\left(t_{i}\right)}\right]^{2}}$

Step 2: For $i=i+1$, 


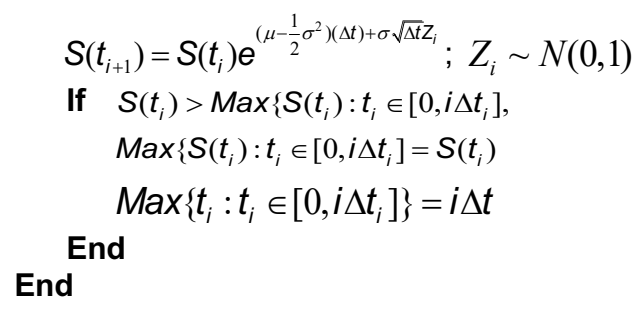

Step 3: If $i \Delta t=T$, stop

otherwise repeat Step 2

End

Procedure 2: forecasting the stock prices by Fourier's series

Let $f(t)$ of stock prices on time interval $t \in[0, T]$.

Step 1: initial values

$$
\begin{aligned}
& k=M, \text { for some } M \\
& n=N, \text { for some } N \\
& L=l, \text { for some } l
\end{aligned}
$$

Step 2: Calculate coefficients of Fourier's series

$$
\begin{aligned}
& a_{0}=\frac{1}{k} \sum_{t=1}^{k} f(t) \\
& a_{n}=\frac{2}{k} \sum_{t=1}^{k} f(t) \cos \left(\frac{2 n \pi t}{L}\right) \\
& b_{n}=\frac{2}{k} \sum_{t=1}^{k} f(t) \sin \left(\frac{2 n \pi t}{L}\right)
\end{aligned}
$$

Step 3: Estimate Fourier's series

$$
\hat{f}(t)=a_{0}+\sum_{n=1}^{N}\left[a_{n} \cos \left(\frac{2 n \pi t}{L}\right)+b_{n} \sin \left(\frac{2 n \pi t}{L}\right)\right]
$$

Procedure 3: forecasting the stock prices by Cauchy initial value problem Let $y\left(x_{t_{i}}\right)$ of stock prices on time interval $t_{i} \in[0, T]$.

Step 1: Calculate initial values

$$
\begin{aligned}
& h=t_{i+1}-t_{i} \\
& a=\frac{1}{h} \ln \left(\frac{y\left(x_{t_{i}}\right)}{y\left(x_{t_{i-1}}\right)}\right)
\end{aligned}
$$

Step 2: Calculate

$$
\begin{aligned}
& f\left(t_{i}, y\left(x_{t_{i}}\right)\right)=a y\left(x_{t_{i}}\right) \\
& f^{\prime}\left(t_{i}, y\left(x_{t_{i}}\right)\right)=a y^{\prime}\left(t_{i}\right)=a^{2} y\left(x_{t_{i}}\right) \\
& f^{\prime \prime}\left(t_{i}, y\left(x_{t_{i}}\right)\right)=a^{2} y^{\prime}\left(t_{i}\right)=a^{3} y\left(x_{t_{i}}\right) \\
& v=\frac{f^{\prime \prime}\left(t_{i}, y\left(x_{t_{i}}\right)\right)}{f^{\prime}\left(t_{i}, y\left(x_{t_{i}}\right)\right)} \\
& Q=\frac{f^{\prime}\left(t_{i}, y\left(x_{t_{i}}\right)\right)-f^{\prime \prime}\left(t_{i}, y\left(x_{t_{i}}\right)\right)}{(1-v) v^{2} e^{v t_{i}}} \\
& b=f\left(t_{i}, y\left(x_{t_{i}}\right)\right)-\frac{f^{\prime}\left(t_{i}, y\left(x_{t_{i}}\right)\right)}{v}
\end{aligned}
$$

Step 3: Estimate solutions of Cauchy initial value problems

$$
y\left(x_{t_{i+1}}\right)=y\left(x_{t_{i}}\right)+b h+Q e^{v t_{i}}\left(e^{v h}-1\right), \text { for } i=1,2,3, \ldots
$$

Forecasting and its Error Analysis 
In this research, the better method for forecasting is measured in minimum value of Root Mean Squared Percentage Error (RMSPE). The formula for RMSPE can be defined as

$$
R M S P E=\sqrt{\frac{1}{n} \sum_{t=1}^{n}\left(\frac{x_{t}-\hat{x}_{t}}{x_{t}}\right)^{2}}
$$

where $x_{t}$ is an observation, $\hat{x}_{t}$ is a predicted value.

The criteria for decision the performance of forecasting model as follows:

Table 1. Measurement of forecasting performance.

\begin{tabular}{ll}
\hline RMSPE & Forecasting Accuracy \\
\hline $0 \% \leq R M S P E \leq 5 \%$ & Highly accurate \\
$5 \%<R M S P E \leq 10 \%$ & Fairly accurate \\
$10 \%<R M S P E \leq 20 \%$ & Reasonably accurate \\
$R M S P E>20 \%$ & Unreasonably accurate \\
\hline
\end{tabular}

\section{Confidence Intervals for predicted stock prices}

The $95 \%$ confidence intervals for predicted stock prices are based on any random variable $X \sim N\left(\mu, \sigma^{2}\right)$. Standardization of $x$ to standard normal is applied by transformation

$Z=\frac{X-\mu}{\sigma} \sim N(0,1)$

$\mathbf{P}\left(-Z_{\alpha / 2} \leq Z \leq Z_{\alpha / 2}\right)=1-\alpha ; \alpha=0.05$

$\mathbf{P}\left(-Z_{\alpha / 2} \leq \frac{X-\mu}{\sigma} \leq Z_{\alpha / 2}\right)=1-\alpha ; \alpha=0.05, Z_{\alpha / 2}=1.96$

$\mathbf{P}(\mu-1.96 \sigma \leq X \leq \mu+1.96 \sigma)=0.95$

So, $[\mu-1.96 \sigma, \mu+1.96 \sigma]$ is the $95 \%$ confidence interval for predicted stock prices.

Let $S(t)=S_{0} e^{\left(\mu-\frac{1}{2} \sigma^{2}\right) t+\sigma \sqrt{t} Z} ; Z \sim N(0,1)$ be stock prices movement simulated by Geometric Brownian motion. The $95 \%$ confidence interval for $S(t)$ is

$\left[S_{0} e^{\left(\mu-\frac{1}{2} \sigma^{2}\right) t-1.96 \sigma \sqrt{t}}, S_{0} e^{\left(\mu-\frac{1}{2} \sigma^{2}\right) t+1.96 \sigma \sqrt{t}}\right]$

Let $\hat{f}(t)=a_{0}+\sum_{n=1}^{N}\left[a_{n} \cos \left(\frac{2 n \pi t}{L}\right)+b_{n} \sin \left(\frac{2 n \pi t}{L}\right)\right]$ be stock prices movement simulated by Fourier's series.

The $95 \%$ confidence interval for $\hat{f}(t)$ is

$\left[a_{0}-1.96 \sqrt{\operatorname{var}(\hat{f}(t))}, a_{0}+1.96 \sqrt{\operatorname{var}(\hat{f}(t))}\right]$

Let $y\left(x_{t}\right)=y\left(x_{t}\right)+b h+Q e^{v t}\left(e^{v h}-1\right)$ be stock prices movement simulated by Cauchy initial value problem. The $95 \%$ confidence interval for $y\left(x_{t}\right)$ is

$\left[\mathbf{E}\left(y\left(x_{t}\right)\right)-1.96 \sqrt{\operatorname{var}\left(y\left(x_{t}\right)\right)}, \mathbf{E}\left(y\left(x_{t}\right)\right)+1.96 \sqrt{\operatorname{var}\left(y\left(x_{t}\right)\right)}\right]$

\section{Results and discussion}

This section is focused on the results and output analysis from forecasting models based on Geometric Brownian Motion, Fourier's series, and Cauchy initial value problem. Weekly data was sampled from cyclical and non-cyclical stocks on The Stock Exchange of Thailand. The basic statistics, forecasting models, and errors are demonstrated and compared as follows. 


\section{Basic statistics and Beta value of cyclical and non-cyclical stock prices}

Table 2. Basic statistics for cyclical stock price.

\begin{tabular}{lllll}
\hline Descriptive Statistics & CK & ITD & NWR & TTA \\
\hline Mean & 15.89576 & 4.97227 & 0.98778 & 16.40542 \\
Standard Error & 0.32761 & 0.07975 & 0.02502 & 0.29144 \\
Median & 12.39020 & 4.44173 & 0.79000 & 15.73750 \\
Mode & 26.75000 & 5.10000 & 0.50760 & 9.05000 \\
Standard Deviation & 9.21990 & 2.24436 & 0.70419 & 8.20198 \\
Sample Variance & 85.00661 & 5.03717 & 0.49589 & 67.27245 \\
Kurtosis & -1.60955 & -0.50651 & 1.81058 & 1.24307 \\
Skewness & 0.19906 & 0.58974 & 1.35136 & 0.98683 \\
Range & 31.72073 & 10.61630 & 3.85176 & 46.57680 \\
Minimum & 2.02927 & 1.45000 & 0.10450 & 3.60000 \\
Maximum & 33.75000 & 12.06630 & 3.95626 & 50.17680 \\
Sum & 12589.43799 & 3938.03739 & 782.31872 & 12993.08927 \\
Beta & 1.40 & 1.61 & 1.20 & 1.52 \\
\hline
\end{tabular}

Table 3. Basic statistics for non-cyclical stock price.

\begin{tabular}{lllll}
\hline Descriptive Statistics & BDMS & CPALL & CPF & TU \\
\hline Mean & 11.74032 & 33.56777 & 19.53577 & 13.13002 \\
Standard Error & 0.31468 & 0.93906 & 0.39638 & 0.21526 \\
Median & 9.90000 & 34.62500 & 24.40000 & 14.43750 \\
Mode & 25.00000 & 5.40000 & 25.00000 & 6.36762 \\
Standard Deviation & 8.85587 & 26.42747 & 11.15505 & 6.05787 \\
Sample Variance & 78.42651 & 698.41122 & 124.43522 & 36.69780 \\
Kurtosis & -1.58423 & -1.10234 & -1.42453 & -1.51999 \\
Skewness & 0.26629 & 0.38715 & -0.33607 & -0.16547 \\
Range & 25.61000 & 85.80000 & 38.45000 & 19.28458 \\
Minimum & 1.39000 & 2.70000 & 2.80000 & 3.71542 \\
Maximum & 27.00000 & 88.50000 & 41.25000 & 23.00000 \\
Sum & 9298.32999 & 26585.670000 & 15472.33000 & 10398.97735 \\
Beta & 0.63 & 0.91 & 0.90 & 0.94 \\
\hline
\end{tabular}

Table 2 and table 3 showed the basic statistics and Beta value. Obviously, cyclical stock price have the Beta value which is larger than Beta value of non-cyclical stock price.

\section{Geometric Brownian motion for cyclical and non-cyclical stock prices}

The important parameters for Geometric Brownian motion are drift and volatility of cyclical stock price and non-cyclical stock price showed in Table 4.

As shown is Table 4, the volatilities of cyclical stock prices are higher than the volatilities of non-cyclical stock prices. However, the drift of cyclical stock prices are lower than the drift of non-cyclical stock prices. 
Table 4. Parameters of Geometric Brownian motion model of cyclical and non-cyclical stock prices.

\begin{tabular}{lcccc}
\hline \multicolumn{5}{c}{ Cyclical Stock Prices } \\
\hline CK & ITD & NWR & TTA \\
\hline Drift & 0.003365388 & 0.001574302 & 0.003599534 & 0.000269615 \\
\hline \multicolumn{5}{c}{ Non-cyclical Stock Prices } \\
\hline BDMS & CPALL & CPF & TU \\
Drift & 0.062700335 & 0.069334042 & 0.085138181 & 0.061015614 \\
Volatility & 0.00499242 & 0.005990429 & 0.004142155 & 0.002724249 \\
\hline
\end{tabular}

\section{Fourier's series for cyclical and non-cyclical stock prices}

The Fourier's series estimations for the cyclical stocks are as follows:

1. $\mathrm{CH}$. Karnchang Public Company Limited (stock name: $\mathrm{CK}$ )

Fourier's series for estimation of CK stock price is

$\hat{f}(t)=13.37919+0.04892 \cos (t)+0.03867 \sin (t)-0.03402 \cos (2 t)$

$+0.00371 \sin (2 t)+0.00879 \cos (3 t)-0.12322 \sin (3 t)$

2. Italian-Thai Development Corporation Limited (stock name: ITD)

Fourier's series for estimation of ITD stock price is

$\hat{f}(t)=5.45219-0.02879 \cos (t)+0.00714 \sin (t)-0.00202 \cos (2 t)$

$-0.00129 \sin (2 t)+0.00207 \cos (3 t)-0.02965 \sin (3 t)+\ldots$

3. Nawarat Patanakarn Public Company Limited (stock name: NWR)

Fourier's series for estimation of NWR stock price is

$\hat{f}(t)=1.00335-0.00159 \cos (t)-0.00279 \sin (t)+0.00156 \cos (2 t)$

$+0.00201 \sin (2 t)+0.00172 \cos (3 t)-0.00743 \sin (3 t)$

4. Thoresen Thai Agencies Public Company Limited (stock name: TTA)

Fourier's series for estimation of TTA stock price is

$\hat{f}(t)=18.76881-0.06670 \cos (t)-0.01167 \sin (t)+0.02383 \cos (2 t)$

$+0.02543 \sin (2 t)-0.05415 \cos (3 t)-0.00370 \sin (3 t)$

The Fourier's series estimations for the non - cyclical stocks are as follows:

1. Bangkok Hospital (stock name: BDMS)

Fourier's series for estimation of BDMS stock price is

$\hat{f}(t)=8.76663+0.07365 \cos (t)+0.03642 \sin (t)-0.02938 \cos (2 t)$

$+0.01588 \sin (2 t)+0.01877 \cos (3 t)-0.05776 \sin (3 t)$

2. CP All Public Company Limited (stock name: CPALL)

Fourier's series for estimation of CPALL stock price is

$\hat{f}(t)=23.32368+0.25969 \cos (t)+0.13332 \sin (t)-0.06308 \cos (2 t)$

$+0.04283 \sin (2 t)+0.05293 \cos (3 t)-0.14306 \sin (3 t)$

3. Charoen Pokphand Foods Public Company Limited (stock name: CPF)

Fourier's series for estimation of CPF stock price is

$\hat{f}(t)=17.82108+0.07190 \cos (t)+0.04877 \sin (t)-0.02967 \cos (2 t)$

$+0.02927 \sin (2 t)+0.01995 \cos (3 t)-0.10354 \sin (3 t)$

4. Thai Union Group Public Company Limited (stock name: TU)

Fourier's series for estimation of TU stock price is

$\hat{f}(t)=11.81933+0.06798 \cos (t)+0.05679 \sin (t)-0.05549 \cos (2 t)$

$-0.01414 \sin (2 t)+0.00576 \cos (3 t)-0.05978 \sin (3 t)$ 


\section{Comparison of Results among Geometric Brownian motion,} Fourier's series, and Cauchy initial value problem

Next, the graphs of actual stock price, predicted stock price by Geometric Brownian motion, Fourier's series, and Cauchy initial value problem are plotted for comparison of the results. Also, the comparison of the errors are provided as follows.
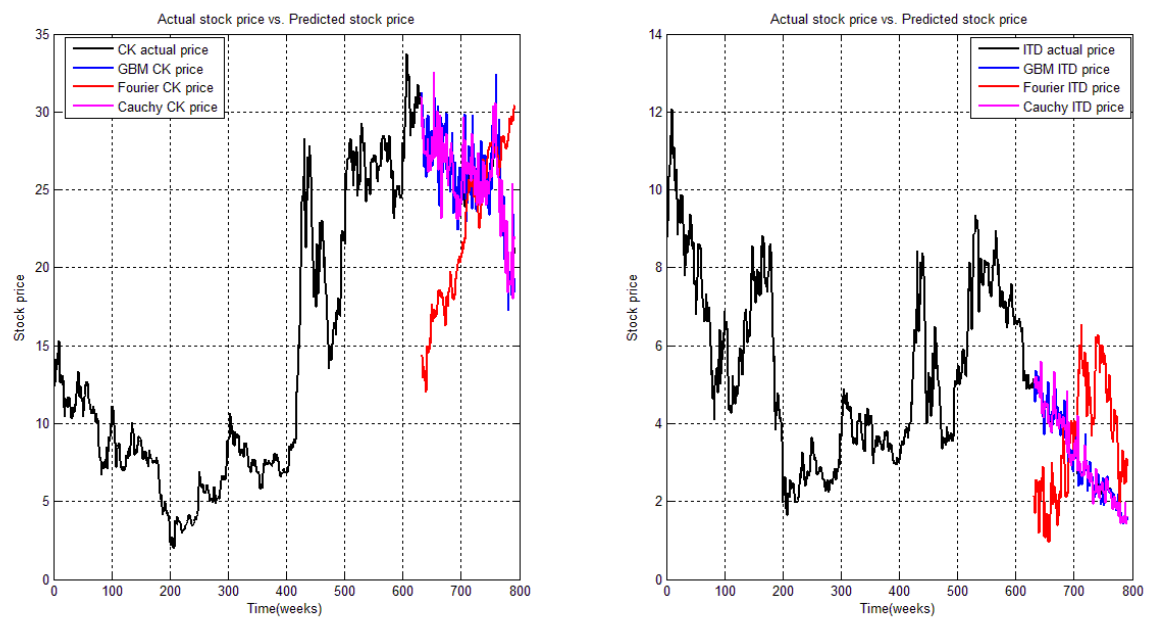

Figure 1. Cyclical CK and ITD stock prices.
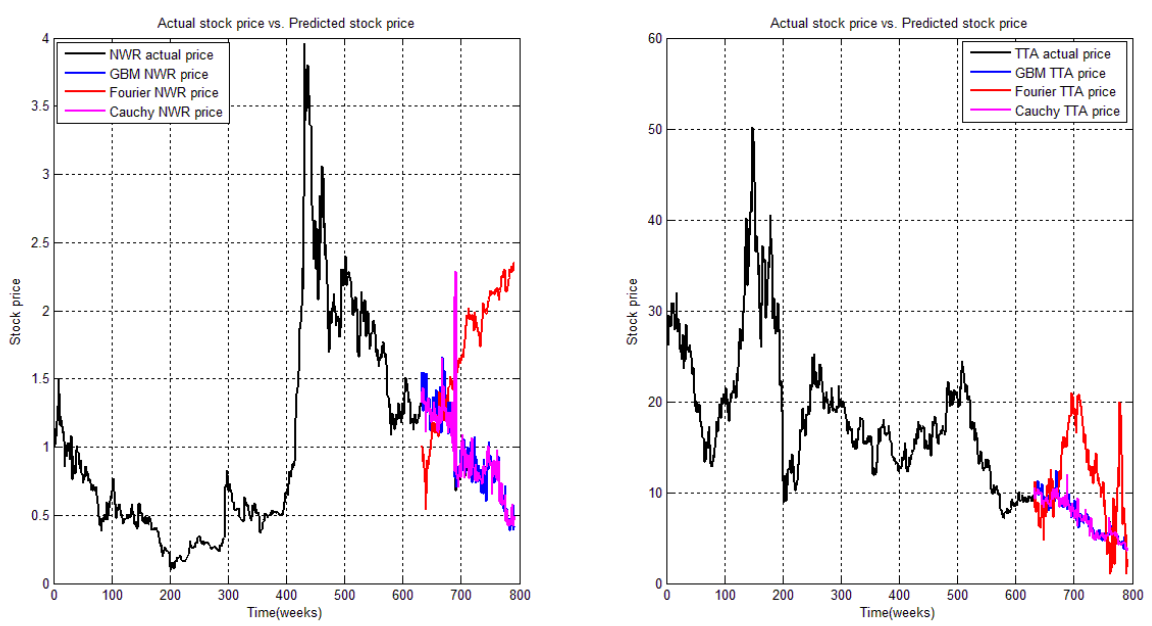

Figure 2. Cyclical NWR and TTA stock prices.

According to Figure 1 and Figure 2, the graphs of cyclical stock price stock prices were plotted to compare the predicted stock prices with actual prices. Obviously, predicted stock prices by both Geometric Brownian motion and Cauchy initial value problems approached the actual stock prices while predicted stock prices by Fourier's series fluctuated and trended around the actual prices. 

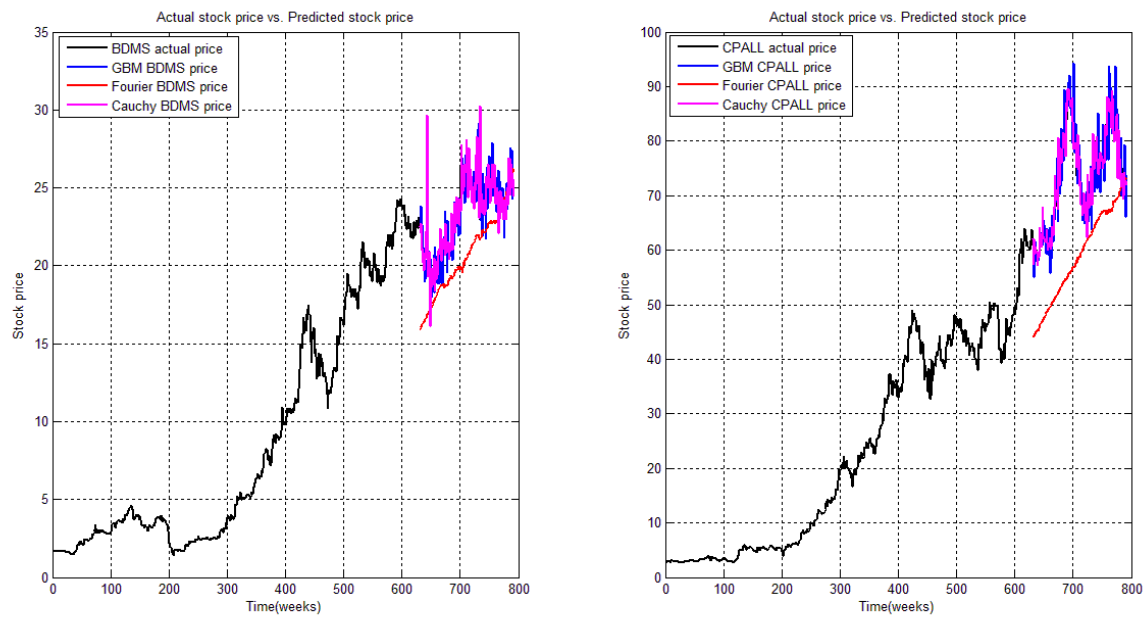

Figure 3 Non-cyclical BDMS and CPALL stock prices
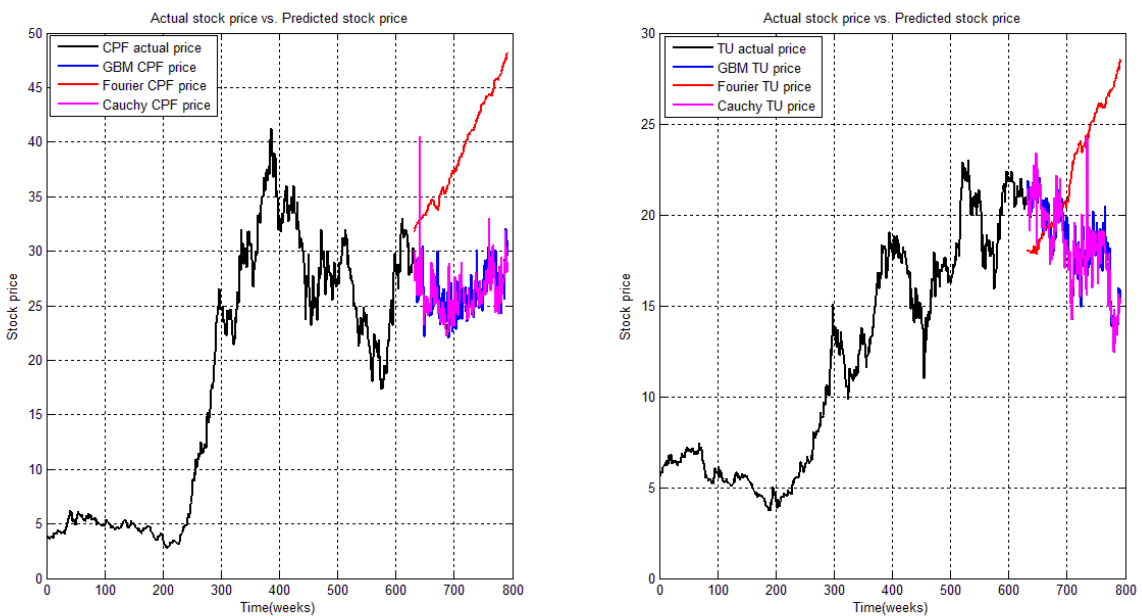

Figure 4. Non-cyclical CPF and TU stock prices.

According to Figure 3 and Figure 4, the graphs of non-cyclical stock price prices were plotted to compare the predicted stock prices with actual prices. It found that predicted stock prices by both Geometric Brownian motion and Cauchy initial value problems changed slightly around the actual stock prices while predicted stock prices by Fourier's series trended down and trended up the actual prices. For comparison of the forecasting errors, the RMSPE are adopted to compare amongst the forecasting methods as follows.

As can be seen from Table 5, the performances of forecasting models on cyclical and non-cyclical stock prices using Geometric Brownian motion (RMSPE $=0.007082387$ and 0.002666017 ), Fourier's series (RMSPE=1.223996238 and 0.062644341), and Cauchy initial value problem (RMSPE $=0.014683423$ and 0.071304403 ) are in highly accurate level because all averages of RMSPE on each of the methods are less than $5 \%$. Furthermore, compared each of the methods between cyclical and non-cyclical stock prices, Geometric Brownian motion and Fourier's series on non-cyclical stock prices are better performances than on cyclical stock prices because the averages on the both methods are less than on non-cyclical stock prices. Whereas, Cauchy initial value problem method on cyclical stock prices is a better performance than on non-cyclical stock prices because the average of Cauchy initial value problem method on cyclical stock prices is lower than on non-cyclical stock prices. 
Table 5. RMSPE for weekly forecasting on stock prices.

\begin{tabular}{lcccc}
\hline \multirow{2}{*}{ Stock name } & \multicolumn{4}{c}{ RMSPE } \\
& GBM & Courier & Cauchy & Average \\
\hline CK & 0.004115606 & 0.088262533 & 0.00154409 & 0.031307410 \\
ITD & 0.006748499 & 0.722771263 & 0.005597916 & 0.245039226 \\
NWR & 0.011202130 & 2.936043833 & 0.047366956 & 0.998204306 \\
TTA & 0.006263311 & 1.148907321 & 0.004224731 & 0.386465121 \\
Average & 0.007082387 & 1.223996238 & 0.014683423 \\
\hline \multicolumn{4}{c}{ RMSPE } \\
Stock name & GBM & Non-cyclical stock prices & \\
& 0.002693709 & 0.018187255 & 0.002626916 & 0.007835960 \\
\hline BDMS & 0.002556077 & 0.046442753 & 0.000631458 & 0.016543429 \\
CPALL & 0.003002090 & 0.002173560 & 0.279774346 & 0.094983332 \\
CPF & 0.002412193 & 0.183773796 & 0.002184891 & 0.062790293 \\
TU & 0.002666017 & 0.062644341 & 0.071304403 \\
Average & \multicolumn{3}{c}{} \\
\hline
\end{tabular}

Predicted stock prices and its confidence intervals

Confidence intervals for predicted stock prices are shown as in Table 6 and Table 7.

Table 6. Confidence Interval for prediction of cyclical stock prices.

\begin{tabular}{ccccc}
\hline Week & Stock Name & Forecasting Model & Prediction & $95 \%$ Confidence Interval \\
\hline CK & GBM & 19.75268455 & 14.00848551 & 25.49688 \\
& Fourier & 29.13458677 & 19.24865666 & 39.02052 \\
& Cauchy & 19.200001 & 13.98477899 & 24.41522 \\
& GBM & 1.520496099 & 0 & 3.672866 \\
& ITD & 2.745093166 & 0 & 5.618781 \\
& Fourier & 1.5101466 & 0 & 3.64157 \\
& Cauchy & 0.423845786 & 0 & 1.009666 \\
& GWM & 2.242715514 & 1.341089516 & 3.144342 \\
& Fourier & 0.45042715 & 0 & 1.055447 \\
& Cauchy & 4.526042438 & 0.518776251 & 8.533309 \\
& GBM & 14.1872274 & 4.508681921 & 23.86577 \\
& FTA & 4.185922451 & 0.243597006 & 8.128248 \\
\hline & Courier & 19.15820636 & 13.41400732 & 24.90241 \\
& GBM & 29.59350724 & 19.70757713 & 39.47944 \\
& GK & 19 & 13.78477799 & 24.21522 \\
& Fourier & 1.520272688 & 0 & 3.672643 \\
& Cauchy & 3.316416678 & 0.442728934 & 6.190104 \\
& GBM & 1.6 & 0 & 3.731423 \\
& Fourier & 0.3882982 & 0.974119 \\
& Cauchy & 2.299851718 & 1.39822572 & 3.201478 \\
& GBM & 0.47 & 0 & 1.075019 \\
& Fourier & 4.224629355 & 0.217363168 & 8.231896 \\
& Cauchy & 12.38494158 & 2.706396097 & 22.06349 \\
& GBM & 4.464304723 & 0.521979279 & 8.40663 \\
\hline
\end{tabular}


Table 7. Confidence Interval for prediction of non-cyclical stock prices.

\begin{tabular}{|c|c|c|c|c|c|}
\hline \multirow{2}{*}{$\frac{\text { Week }}{783}$} & \multirow{2}{*}{$\begin{array}{c}\text { Stock Name } \\
\text { BDMS }\end{array}$} & \multirow{2}{*}{$\begin{array}{c}\text { Forecasting Model } \\
\text { GBM }\end{array}$} & \multirow{2}{*}{$\begin{array}{c}\text { Prediction } \\
24.55002961\end{array}$} & \multicolumn{2}{|c|}{ 95\% Confidence Interval } \\
\hline & & & & 20.35596202 & 30.30006923 \\
\hline & & Fourier & 25.18620771 & 20.01842852 & 30.3539869 \\
\hline & & Cauchy & 26.90009313 & 22.07813475 & 31.72205151 \\
\hline & CPALL & GBM & 80.52442125 & 61.93928641 & 99.10955608 \\
\hline & & Fourier & 71.65794137 & 55.08655763 & 88.22932511 \\
\hline & & Cauchy & 71.60115722 & 54.32183176 & 88.88048268 \\
\hline & CPF & GBM & 27.06420501 & 23.05781251 & 31.0705975 \\
\hline & & Fourier & 27.004773 & 37.18660203 & 56.24998939 \\
\hline & & Cauchy & 27.004773 & 22.51695055 & 31.49259545 \\
\hline & TU & GBM & 13.8255846 & 9.492567774 & 18.15860143 \\
\hline & & Fourier & 14.34223888 & 20.93571559 & 33.98615502 \\
\hline & & Cauchy & 14.34223888 & 9.93399854 & 18.75047922 \\
\hline 784 & BDMS & GBM & 25.89561593 & 19.577976 & 29.52208321 \\
\hline & & Fourier & 25.5096696 & 20.34189041 & 30.67744879 \\
\hline & & Cauchy & 26 & 21.17804162 & 30.82195838 \\
\hline & CPALL & GBM & 69.98793022 & 51.40279539 & 88.57306506 \\
\hline & & Fourier & 71.92825662 & 55.35687289 & 88.49964036 \\
\hline & & Cauchy & 72 & 54.72067454 & 89.27932546 \\
\hline & CPF & GBM & 26.66821389 & 22.6618214 & 30.67460639 \\
\hline & & Fourier & 46.96292612 & 37.43123244 & 56.49461979 \\
\hline & & Cauchy & 27 & 22.51217755 & 31.48782245 \\
\hline & TU & GBM & 13.6274064 & 9.294389571 & 17.96042323 \\
\hline & & Fourier & 27.52170178 & 20.99648207 & 34.04692149 \\
\hline & & Cauchy & 14 & 9.59175966 & 18.40824034 \\
\hline
\end{tabular}

\section{Conclusions}

In this research, the methods for forecasting stock prices in a weekly period consisting of cyclical and non-cyclical stock prices collected on the Stock Exchange of Thailand were proposed. The comparison of forecasting error and accuracy of forecasting models based on RMSPE were demonstrated and compared. Also, the confidence intervals of predicted stock prices were proposed. Based on the sample stock prices, it was found that the cyclical stock prices take the higher risk than non-cyclical stock prices because the Beta values of cyclical stock prices are greater than non-cyclical stock prices. The results showed that Geometric Brownian motion and Fourier's series performed on non-cyclical stock prices better than on cyclical stock prices based on RMSPE which is a highly accurate level. While Cauchy initial value problem performed on cyclical stock prices better than on non-cyclical stock prices based on RMSPE which is also a highly accurate level. Overall, all averages on the methods for forecasting provided a good performance because all of the RMSPEs were less than $5 \%$ which is highly accurate. Furthermore, compared amongst Geometric Brownian motion, Fourier's series, and Cauchy initial value problem, the result showed that Geometric Brownian motion is a good performance method for forecasting both cyclic and non-cyclical stock prices because RMSPE as the error between actual and predicted stock prices is minimum. The benefits of this research are the efficient ways for making decisions for analysts working such as marketing research in forecasting sales, prediction on future contract of commodities in ingredient companies, as well as investment on securities in the financial sector, etc. However, future research works can be extended to other asset movements apart from stock prices and combined forecasting should be considered for future research works. 


\section{Conflicts of interest}

We declare that we have no conflict of interest.

\section{References}

[1] G. Box and G. Jenkins, "Time series analysis: forecasting and control," San Francisco: Holden-Day; 1970.

[2] J. Contreras, R, Espinola, F, Nogales and A. Conejo, "ARIMA Models to Predict Next day Electricity prices," IEEE Transactions on Power Systems, vol. 18, no.3, pp. 1014- 1020, 2003.

[3] E. Weiss, "Forecasting commodity prices using ARIMA," Technical Analysis of Stocks \& Commodities, vol. 18, no. 1, pp. 18-19, 2000.

[4] A. Rahman and M. Hasan, "Modeling and Forecasting of Carbon Dioxide Emissions in Bangladesh Using Autoregressive Integrated Moving Average (ARIMA) Models," Open Journal of Statistics, vol. 7, pp. 560-566, 2007.

[5] N. Thabani, O. Hamadziripi and M. Chipo, "Modelling and forecasting carbon dioxide emissions in China using autoregressive integrated moving average (ARIMA) models," International Journal of Multidisciplinary Research, vol. 5, no. 4, pp. 2455-3662, 2019.

[6] S. Ketut and J. Miftahul, "Forecasting Model Selection of Curly Red Chili Price at Retail Level," Indonesian Journal of Agricultural Research, vol. 2, no.1, pp. 1-12, 2019.

[7] R. Anokye , E. Acheampong, I. Owusu and E. Obang, "Time series analysis of malaria in Kumasi: Using ARIMA models to forecast future incidence," Cogent Social Sciences, vol. 4, no. 1, pp. 1-13, 2018.

[8] K. Divya, S. Rajeswari, D. Bhavani and P. Sumathi, "Forecasting Monthly Prices of Bengalgram in Selected Markets of Andhra Pradesh," International Journal of Research in Agricultural Sciences, vol. 4, no. 4, pp. 23483997, 2017.

[9] S. Nop, "Trading Gold Future with ARIMA-GARCH model," Thai Journal of Mathematics, Special Issue (2018), pp. 227-238, 2017.

[10] H. Pham and B. Yang, "Estimation and forecasting of machine health condition using ARMA/GARCH model," Mechanical Systems and Signal Processing, vol. 24, pp. 546-558, 2010.

[11] H. Liu and J. Shi. "Applying ARMA-GARCH approaches to forecasting short-term electricity prices," Energy Economics, vol. 37, pp. 152-166, 2013.

[12] B. Zou, D. He and Z. Sun, "Traffic Modeling and Prediction using ARIMA/GARCH Model in Modeling and Simulation Tools for Emerging Telecommunication Networks," Boston: Springer; 2006.

[13] D. Chaido, "The Performance of Hybrid ARIMA-GARCH Modeling and Forecasting Oil Price," International Journal of Energy Economics and Policy, vol. 8, no. 3, pp. 14-21, 2018.

[14] P. Pai and C. Lin, "A hybrid ARIMA and support vector machines model in stock price forecasting," Omega, vol. 33, pp. 497-505, 2005.

[15] G. Zhang, "Time series forecasting using a hybrid ARIMA and neural network model," Neuro computing, vol. 50, no. 50, pp. 159-175, 2003.

[16] M. Fang M, H. Gwo, C. Hsiao and J. Benjamin, "Fuzzy ARIMA model for forecasting the foreign exchange market," Fuzzy Sets and Systems, vol. 118, no. 1, pp. 9-19, 2001.

[17] T. Sheida, K. Mehdi and B. Mehdi, "A hybrid probabilistic fuzzy ARIMA model for consumption forecasting in commodity markets," Economic Analysis and Policy, vol. 58, pp. 22-31, 2018.

[18] M. Fang and H. Gwo, "A fuzzy seasonal ARIMA model for forecasting," Fuzzy Sets and Systems, vol. 126, pp. 367-376, 2002.

[19] The Stock Exchange of Thailand, "Securities Analysis using Fundamental Analysis," $4^{\text {th }}$ edition Bangkok: The Stock Exchange of Thailand; 2002.

[20] D. Higham, "An Introduction to Financial Option Valuation: Mathematics, Stochastics and Computation," UK: Cambridge University Press; 2004.

[21] P. O'Neil, "Advanced Engineering Mathematics," $7^{\text {th }}$ edition, Boston: PWS Publishing; 2011.

[22] L. Marcela, "The analysis of the commodity price forecasting success considering different numerical models sensitivity to prognosis error," Acta Logistica, vol. 3, no. 4, pp. 7-15, 2016. 